\title{
Téoros
}

Revue de recherche en tourisme

\section{Le golf en Estrie}

\section{François Giasson}

Volume 16, numéro 1, printemps 1997

La région touristique de l'Estrie / Cantons-de-l'Est

URI : https://id.erudit.org/iderudit/1074926ar

DOI : https://doi.org/10.7202/1074926ar

Aller au sommaire du numéro

\section{Éditeur(s)}

Université du Québec à Montréal

\section{ISSN}

0712-8657 (imprimé)

1923-2705 (numérique)

Découvrir la revue

\section{Citer cet article}

Giasson, F. (1997). Le golf en Estrie. Téoros, 16(1), 38-45.

https://doi.org/10.7202/1074926ar d'utilisation que vous pouvez consulter en ligne.

https://apropos.erudit.org/fr/usagers/politique-dutilisation/ 


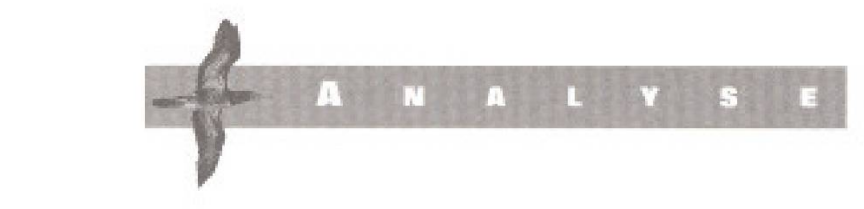

\section{Le GOLF EN ESTRIE}

François Giasson, géographe MRC de La Haute-Yamaska, Granby

Le golf est devenu whe activite wes importante au Quebec. Selon notre etude, 703000 personnes (dont 89 o0o membres) jouen au golf au moins une fois par année sur les 340 terrains de golf quebecois.

\section{HISTORIQUE DU GOLF EN ESTRIE}

L'Estrie fut l'une des premières régions du Québec à être prise d'assaut par de riches industriels américains, ayant des affinités avec les Loyalistes qui profitèrent de leurs vacances pour faire construire des terrains de golf, Ces terrains à l'époque ntétaient pas des terrains de challenge mais des terrains moyens, construits pour le loisir de ces riches Américains tels les Ford, les Rockefeller, les Flagler, les Vanderbilt, etc.

The Old Lennox golf Club (1897), appartenant aujourd'hui à l'Université Bishop. fut le premier terrain construit en Estrie tandis que le club de grolf Waterville (1903) fut le deuxième. Le club de golf Miner, nom d'une riche famille industrielle anglaise dont les racines sont toujours existantes à Granby, fut le troisième club existant. Le club de golf appartient toujours à la famille Miner qui loue le terrain aux membres du club. Le Hermitage Golf Club (1912) fut construit pendant le première guerre mondiale, a eté incendie et reconsiruit en 1928. II en fut de même pour le club de golf de Cowansville (1924) qui a áté rasế par le feu en 1960 puis reconstruit en 1964. Le club de golf de Knowlton (1920), le
Dufferin-Heights (1923). le Massawippi à North Hatley (1924), le Country Club de Sherbrooke (stappelant autrefois le Saint-Francois) (1926) ont tous des noms qui évoquent un passể américain. Même si les terrains de golf du passé sont riches en histoire, seul le Country Club de Sherbrooke avait 18 trous à cette époque. Ces huit terrains sont sans équivoque les plus vieux en Estrie et font partie des 20 plus vieux au Québec tout comme le Royal Montréal (1873), le Royal Québec (1891), le Ki-Wi-Eb (1923), le Manoir Richelieu (1924), le Grand-Mère, le Beaconsfield (1904), Laval-sur-le-Lac (1916) et le Drummondville. L'Estrie a été le chateau fort des terrains de golf de l'Amérique à cette ếpoque.

Depuis ces années, il n'y a eu que deux ouvertures de terrains de golf en Estrie avant les années 1960 , soit le club de golf du Mont-Orford (1939) et le club de golf Farnham (1959). Un boom économique favorable a permis la réalisation de plus de 30 autres terrains jusqu'à aujourd' hui. Ce chiffre va toujours s'accroitre, on peut en être assuré. Ce n'est que vers les années 1950 que les francophones sont devenus de grands adeptes de golf. Auparavant, e'était un sport d'anglophones.

\section{L'ESTRIE, UNE RÉGION DE GOLF UNIQUE AU QUÉBEC}

Le golf est un produit de consommation important en Estrie. Cette région est troisième quant au nombre de parcours de golf (42) apres la Montérégie (62) et les Laurentides (44). Si ces deux dernières régions contiennent beaucoup de parcours, c'est parce qu'elles accueillent en grande quantite les golfeurs de Montréal. Plus de $11,8 \%$ des 340 terrains de golf du Québec sont situés en Estrie.

Les 42 terrains de golf sont dispersés à l'intérieur des 9 municipalités régionales de comté (MRC) de l'Estric. Avec près de $25 \%$, la MRC de La Haute-Yamaska est celle qui compte le plus de terrains de golf en Estrie (voir tableau 1).

De par sa situation géographique australe, l'Estrie jouit d'une température de quelques degrés plus chande qu'ailleurs en province. Certains touristes préferent l'Estric aux Laurentides du fait qu'il n' $y$ a pas de moustiques. Ces touristes sont nombreux en région car la majorité des terrains de golf de l'Estrie sont moins occupés que les terrains des autres régions du Québec.

L'Estric se classe troisième au Québec quant au nombre d'habitants par tertain de golf. Avec une moyenne de 9607 habitants par terrain de golf, ce taux est deux fois moins élevé que la moyenne québécoise. Seules les Laurentides (9 438 habitants) et la Gaspesie (7 859 habitants) sont plus accessibles à une clientèle locale et régionale. Par le fait même, la région de Montréal ( 149938 habitants) et la région de Laval sont les moins bien 
représentées au Québec quant au nombre d'habitant par terrain de golf (voir tableau 2).

En théorie, tout porte à croire qu'il y a maintenant trop de terrains de golf pour répondre à la demande. Selon la National Golf Fondation, la situation la plus adequate est de posséder un terrain de golf par 25000 habitants. En Estrie, ce nombre est d'un terrain de golf pour 9607 habitants. Le flux de villégiateurs, d'excursionnistes et de touristes de Montréal et la rive-sud régularise la situation en occupant davantage les terrains de golf dans les MRC de La HauteYamaska, Brome-Missisquoi et Memphrémagog (voir tableau 3).

\section{FAITS SAILLANTS SUR LE GOLF DANS LA RÉGION TOURISTIQUE DE L'ESTRIE}

- II en coûte moins de 1000 S pour devenir membre dans 31 des 40 terrains de golf de l'Estrie. La moyenne des couts de cotisation dans les 40 clubs de golf de l'Estrie est de $767 \mathrm{~S}$ annuellement:

- $95 \%$ des parcours, soit 38 sur 40 , sont ouverts au public. Ce taux propulse l'Estrie, en tête du palmarès des régions du Québec face à son ouverture à une clientele itinérante:

- Il se joue annuellement 840000 parties de golf en Estrie, soit une moyenne de 21 000 par club de golf. 237695 parties de golf sont jouées par des golfeurs de l'extérieur de l'Estrie soit $28,3 \%$ des parties totales;

- 32,5\% des terrains acceptent des joueurs sans réservation et ce, même les fins de semaine. Les terrains de golf de l'Estrie sont de plus en plus accessibles à une clientèle impulsive;

- $37,5 \%$ des terrains de golf (15) sont situés à moins d'une heure de roule de Montrúal:

- Le club de golf Venise à Magog est celui qui accueille le plus de parties de golf dans une année avec 58000 . A l'inverse, le club de golf le MontPlaisit des Trois-Lacs n'accueille que 500 parties:

- La longueur de jeu totale en Estrie est de 207725 verges:
- 29 terrains possèdent au moins une salle de réception;

- Il s'est réalisé 1275 tournois de golf sur le territoire de l'Estrie totalisant près de 91000 golfeurs participants;

- Le golf emploie, de façon directe en Estrie, 976 personnes, celle-ci étant pour la plupart des travailleurs saisonniers.

\section{LES CARACTÉRISTIQUES DES GOLFEURS UOUANT} SUR LES PARCOURS DE L'ESTRIE)

\section{Le nombre de joueurs}

On dénombre près de 71000 golfeurs sur les 40 terrains de l"Estrie. De ce nombre, seulement 33298 personnes jouent cinq parties ou plus durant l'année et 9000 sont membres (voir tableau 4).

- Les 33298 golfeurs (jouant plus de cinq parties par an) jouent en moyenne 22,2 parties par année.

- Plus de la moitié de la clientèle de golf, soit 37750 participants, jouent moins de 5 parties par annees. On ne peut pas les caractériser de golfeurs car ils jouent si peu souvent. Le terme de participants est plus approprié dans les circonstances. Par contre, les 102000 parties totales que cela occasionne sont des donnés non négligeables et importantes pour les propriétaires de clubs.

Les membres (voir tableau 5)

- Les 40 terrains de golf de l'Estrie comptent 8960 membres soit pres de $10 \%$ des membres québécois.

- Les membres représentent 12,6\% de l'ensemble des participants mais comptent pour $42,9 \%$ des parties totales des terrains de golf de l'Estrie.

- Les membres jouent en moyenne 40,3 parties annuellement. Certains golfeurs jouent jusqu'à 150 parties par année ếtant donné qu'ils refont une deuxième fois le même parcours.

- Le club de golf l'Excellence d'Asbestos est le seul à avoir 500 membres ou plus en Estrie. Les clubs de golf à nombre elevé de membres en Estrie sont peu nombreux si on les compare a certains clubs dans la région de Montréal et de la rive-sud. Des clubs

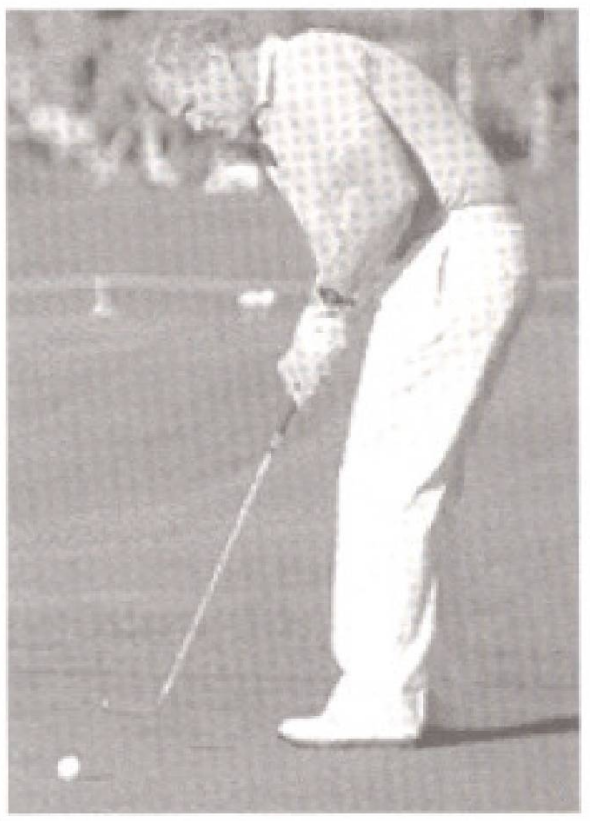

comme Summerlea, La Vallée-duRichelieu, Laval-sur-le-Lac, Hillsdale, Le Blainvillier et Le Royal Montréal par exemple ont plus de 1000 membres chacun.

- Les clubs de golf à saveur touristique ont généralement peu de membres. Les Rochers Bleus de Sutton, le MontOrford, le Lac Lyster, le Manoir des Sables, l'Auberge Bromont et l'Inverugie de Georgeville sont tous des clubs touristiques qui ont $100 \mathrm{mem}$ bres ou moins.

\section{Les golfeurs itinérants}

Ils sont 61887 golfeurs itinérants (les non membres) jouant 480230 parties en Estrie. Ils représentent $87.6 \%$ des golfeurs mais seulement 57,1 \% de l'ensemble des parties de golf, soit une moyenne de 7,8 parties annuellement par golfeur.

\section{Le sexe des joueurs}

D'après notre étude, $65 \%$ du nombre de parties de golf jouées en Estrie en 1995 I'ont été par des personnes du sexe masculin. Ces derniers jouent 546 I85 parties annuellement. Quant aux femmes, elles consomment quand même 293710 parties de golf au total. Il ne faut pas se surprendre du taux de $35 \%$ de participation féminine. Les femmes jouent de plus en plus au golf et cette tendance est nationale. En 1985, elles representaient au Canada $15 \%$ des golfeurs. En 1987, elles Étaient $23,9 \%$ soit une augmentation de 


\begin{tabular}{|c|c|c|}
\hline \multicolumn{3}{|c|}{$\begin{array}{l}\text { Tabieau } 1 \\
\text { Répartition des terrains de golf de I'Estrie touristiques, } 1996\end{array}$} \\
\hline MRC de LEstrie & Nb. de terrains & Liste des terrains en Estrie \\
\hline Asbestas: & 2 & L'excellence of Asbestos et le Mont-Plaisir des Trais-Lacs. \\
\hline Brome-Missisquoi & 6 & $\begin{array}{l}\text { Cowansville, Farnham, Knowlton, lnvemess-Lac Brome, } \\
\text { Les Rochers Bleus et le Domaine Beauregard de Sutton. }\end{array}$ \\
\hline Coaticook & 2 & Costicook et Lac Lyster. \\
\hline La Haute-Yamaska & 10 & $\begin{array}{l}\text { Les Cédres, Granby-Saint-Paul, L'inter golf, Waterloo, } \\
\text { Le Rocher de Raxton Pand, Le Royal Bromant, I'Auberge Bromont, } \\
\text { Du Vieux Village, Granby-Miner et Des Lacs. }\end{array}$ \\
\hline Le Granit & 1 & Lac Meganti: \\
\hline Le Haut-St-Françbiş & 3 & Marbleton, Bury Pen-Y-Bryn et East Angus, \\
\hline Le Val-St-François & 3 & Valcourt, Windsar et Mellbaurne-fichnond. \\
\hline Memphrémaggog & 8 & $\begin{array}{l}\text { Venise, Mont-Orford, Owl's Head, Inveruggie, Hermitage, } \\
\text { Manair des Sables, Dufferin-Heights, Massamipp. }\end{array}$ \\
\hline Sherbrooke & 7 & $\begin{array}{l}\text { Milby, Waterville, Sherbraoke Country Club, Longchamps, } \\
\text { Le Vieux Lennox, Le Gelf de l'Estrie et les Bosquets. }\end{array}$ \\
\hline TOTAL ESTRIE & 42 & $\begin{array}{l}16 \text { parcours de } 9 \text { trous, } 22 \text { parcours de } 18 \text { traus, } 1 \text { parcours de } \\
27 \text { trous, } 2 \text { parcours de } 36 \text { traus et } 1 \text { parcours de } 45 \text { trous. }\end{array}$ \\
\hline
\end{tabular}

\begin{tabular}{|c|c|c|c|}
\hline \multicolumn{5}{|c|}{ Nombre d'habitants par terrain de gall, Esiric, 1951-1996 } \\
\hline Anaés & $\begin{array}{c}\text { Population } \\
\text { I'Estrie }\end{array}$ & $\begin{array}{c}\text { Nombre de terrains } \\
\text { de goll }\end{array}$ & $\begin{array}{c}\text { Nombre d'habitants } \\
\text { par terrain de golf }\end{array}$ \\
\hline 1951 & 265456 & 12 & 22121 \\
\hline 1961 & 309064 & 13 & 23774 \\
\hline 1971 & 335465 & 25 & 13419 \\
\hline 1981 & 362149 & 30 & 12072 \\
\hline 1986 & 366420 & 30 & 12214 \\
\hline 1991 & 387001 & 36 & 10751 \\
\hline 1996 & 403509 & 40 & 9607 \\
\hline
\end{tabular}

\begin{tabular}{|l|c|}
\hline \multicolumn{2}{|c|}{ Tableau 3 } \\
\hline Provenance des joueurs de terrains de golls de l'Estrie, 1996 \\
\hline Origine des joueurs de goll & Pourcentage \\
\hline$\%$ des golfeurs jouant a l'intérieur même de sa MRC. & $44,8 \%$ \\
\hline$\%$ des golfeurs jouant al l'extérieur de sa MRC mais dans une autre MRC de l'Estrie. & $26,9 \%$ \\
\hline$\%$ des golfeurs provenant de leatérieur de l'Estrie & $28,3 \%$ \\
\hline
\end{tabular}

\begin{tabular}{|c|c|c|c|c|}
\hline \multicolumn{5}{|c|}{$\begin{array}{l}\text { Tableau } 4 \\
\text { Les catégories de galfeurs jouant en Estrie, } 1996\end{array}$} \\
\hline Categories de golfeurs & $\begin{array}{l}\text { Nb. de } \\
\text { gplfaurs }\end{array}$ & $\begin{array}{l}\text { go du nombre } \\
\text { de galfeurs }\end{array}$ & $\begin{array}{l}\text { Nb tatal } \\
\text { de parties }\end{array}$ & $\begin{array}{l}\text { \% du nombre } \\
\text { de parties }\end{array}$ \\
\hline $\begin{array}{l}\text { Les participants } \\
\text { (jouant moins de } 5 \text { parties par ant) }\end{array}$ & 37549 & $53,0 \mathrm{H}$ & 101380 & $12,0 \mathrm{y}$ \\
\hline Les non membres 16 a 15 partiest & 17050 & $24,1 \%$ & 170500 & $20,3 \%$ \\
\hline Les non membres |de 16 a 25 parties | & 4406 & $6,2 \%$ & 88120 & $10.5 \%$ \\
\hline Les non membres lde 26 id 50 partiest & 2395 & $3,4 \%$ & 91010 & $10.8 \%$ \\
\hline Las non membres 151 parties et +1 & 487 & $0,7 \%$ & 29220 & $3,5 \%$ \\
\hline Les membres & 8960 & $12,6 \mathrm{~s}$ & 359770 & $42,9 \%$ \\
\hline TOTAUX & 70847 & 100 & 840000 & 100 \\
\hline
\end{tabular}

$60 \%$ en 2 ans. En 1990, ce taux est passé à $27,9 \%$.

- Les terrains courts et assez faciles à jouer sont ceux qui sont les plus appréciés des femmes. Par exemple, les clubs de golf tels les Cèdres, Waterville, Inverugie, MelbourneRichmond, Des Laes, le GranbySaint-Paul sont des clubs qui sont fréquentés par $40 \%$ ou plus de femmes.

- Au contraire, les terrains longs et difficiles n'attirent pas beaucoup de golfeuses. $30 \%$ ou moins de femmes sont présentes à Cowansville, Asbestos, Owl's Head, Mont-Orford et Inverness, terrains reconnus comme étant des parcours longs et ardus).

\section{La langue maternelle}

Plus de 120000 parties de golf sont jouées par des anglophones sur les terrains de l'Estrie. Le taux estrien d'anglophones est de $14,3 \%$. Seulement 15 terrains estriens ont un taux supérieur à la moyenne dont cinq possédant autant ou plus d'anglophones que de francophones, soit Knowlton. Inverugie, DufferinHeights, le Old Lennox et l'Hermitage de Magog.

- La moitié des terrains de golf de l'Estrie comptent moins de $5 \%$ de la clientèle anglophone.

\section{L'âge des golfeurs}

La clientèle de golf́ en Estrie est caractérisée comme étant assez âgée. Le phénomène est d'ailleurs identique en province. En fait. $49 \%$ de la population estrienne est àgée de moins de 35 ans mais ils ne sont que $27 \%$ à jouer au golf. Il est anormal que le taux de joueurs de golf des 18 à 34 ans $(20,1 \%)$ soit inférieur à son taux de représentativité démographique en Estrie $(22,5 \%)$. Cette clientèle, à la fleur de l'âge, devrait représenter au moins $30 \%$ des golfeurs du fait qu'elle recherche généralement à pratiquer de l'activité physique (voir tableau 6).

- Un joueur de golf sur trois est âgé entre 35 et 49 ans.

- Les golfeurs âgés de 50 ans et plus, soit les retraités et les pré-retraités, sont en proportion de $39,7 \%$ dont $36,6 \%$ étant âgés de 55 ans et plus. 
- L'ảge moyen des golfeurs en Estrie est de 42,9 ans.

- Les clubs de golf à saveur locale et régionale ont un taux plus élevé de juniors que les terrains de golf touristiques.

- La moyenne d'age dans les clubs de golf privés de l'Estrie (il n'y en a que 2) est de 53 ans. Par contre, les clubs de golf axés sur les touristes ont pour la plupart une moyenne d'âge se situant en-dessous de la moyenne estrienne (42,9 ans) soit près de 38 ans.

\section{Les juniors}

II $\mathrm{n}^{+} \mathrm{y}$ a que 58300 parties de golf jouées par les juniors en Estrie en 1995. En fait, aucun club ne compte plus de 5000 parties jouếes par les moins de 18 ans. Le club accueillant le plus de parties est le Venise avec 4650 parties... sur les 58000 annuelles. D'après notre étude. la complexité des parcours, la longueur et le prestige de certains clubs de golf ne sont pas des critères qui effraient les golfeurs juniors. Il y a plus de juniors qui jouent au Royal Bromont (le club ayant le coût à la partie le plus élevé en Estrie) qu'à Bury (l'un des 5 terrains les moins dispendieux en Estrie).

Le club de golf qui retrouve la plus forte concentration de juniors est en fait Le Vieux Lennox $(19 \%)$ car il est situé à proximité de l'université Bishop.

$65 \%$ des clubs de golf de l'Estric ont une représentativitét de juniors inférieure à $10 \%$. Un meilleur encadrement, plus de promotion ainsi que plus d'ouverture d'esprit seraient des investissements avantageux pour les propriétaires des clubs de golf car attirer les golfeurs juniors garantirait la clientèle de demain.

\section{Les seniors}

Les golfeurs de 55 ans et plus jouent 307 380 parties de golf annuellement en Estrie, soit $36,6 \%$ des parties. 9 clubs de golf ont une clientèle de seniors à plus de $50 \%$. On les retrouvent en grand nombre dans la majorité des clubs de golf de l'Estrie. Par exemple, les seniors jouent plus de 20000 parties aux Cèdres de Granby. Il se joue également plus de 15000 parties à Cowansville, à Waterloo, à Venise et à l'Hermitage de Magog, soil tous des clubs de golf prestigieux. II $\mathrm{n}^{\prime} \mathrm{y}$ a en fait que deux clubs de golf en Estrie qui possedent une clientèle seniore de moins de $20 \%$.

\section{Les revenus annuels du golfeur}

Les revenus annuels des golfeurs estriens ressemblent étrangement à ceux des golfeurs américains tel que démontré dans l'étude de Cooper's Lybrand en 1990. II n'est plus vrai en 1995 que le golf n'est joué que par des gens riches. Au contraire, notre étude démontre qu'il y a un certain équilibre entre les golfeurs fortunés et les golfeurs moins fortunés. De façon générale, un tiers des golfeurs ont des revenus inférieurs à $30000 \$$, un autre tier gagnent des revenus variant entre 30000 et $50000 \mathrm{~S}$ et un dernier tiers gagnent des revenus supérieurs à $50000 \$$ (voir tableau 7).

\section{L'origine des joueurs de golf}

Press de $72 \%$ des parties de golf sur les terrains de l'Estrie sont consommées par les Estriens, soit 602300 parties par année. Les résidants de la grande région sherbrookoise consomment à eux seuls $27,2 \%$ des parties de golf de l'Estrie (239 400 parties). Quant à la région d'Asbestos, elle représente la région la moins productive en termes de positionnement avec $2.1 \%$ (17 640 rondes) de l'ensemble des parties jouées en Estric (voir tableau 8).

Un total de $28,3 \%$ des parties sont jouées par les joueurs de golf provenant de l'extérieur de la région de l'Estrie. Ce ratio représente 237695 parties par année. Les golfeurs de l'extérieur jouent autant de parties en région que 7 des $9 \mathrm{MRC}$ de I'Estrie regroupées ensemble. $75 \%$ des parties exterieures proviennent de Montréal et de la banlieue;

- 80635 parties $(9,6 \%)$ sont jouées par les gens de la région de Montréal, Laval et Lanaudiere.

- 97430 parties $(11,6 \%)$ sont intimement reliées à la région de la Montérégie.

\section{Nombre d'années de pratique de golf}

La vague la plus forte de nouveaux golfeurs a eu lieu entre 1987 et 1990. En effet, chaque année, $11 \%$ des golfeurs étaient alors des débutants. La croissance du nombre de terrains de golf a suivi la hausse du nombre de joueurs débutants. Six terrains ont été inaugurés à cette epoque. En 1995, le taux de nouveaux joueurs chutait sous la barre des $2,0 \%$, soit le plus bas taux jamais enregistré en Estrie.

\section{L'augmentation du produit de loisir par les golfeurs}

On sait qu'au Québec, il y a eu une perte de 500000 parties annuellement étant donné que le nombre de parties moyen par golfeur est passé de $13,6 \%$ en 1990 à $11,9 \%$ en 1995 .

\section{Les dépenses annuelles pour cette activité de loisir}

Plus de $34,5 \%$ des golfeurs dépensent plus de $1000 \$$ annuellement pour pratiquer ce loisir. Ce montant inclut les droits de jeu, l'achat d'équipement, les voyages de golf, l'après golf, ete (voir tableau 9).

D'après les données du tableau ci-dessus, on peut conclure que le golf est un produit assez dispendieux. II n'y a seulement que $17,5 \%$ des golfeurs qui prétendent que les prix pour jouer au golf sont peu élevés.

\section{La fréquentation des terrains de golf}

$58,6 \%$ des golfeurs estriens jouent sur 2 à 5 terrains différents au cours de leur saison de golf. Par contre, $22,6 \%$ des golfeurs changent continuellement de terrains pour ainsi en connaître davantage sur le produit de golf estrien et québécois. Les $16,3 \%$ des golfeurs pratiquant toujours au même endroit sont pour la plupart des membres.

\section{Les raisons de la pratique du golf}

$47,4 \%$ des golfeurs pratiquent ce loisir pour la santé et la détente. Contrairement aux golfeurs américains, le golf d'affaires est peu représentatif en Estrie. En fait, seulement $4,9 \%$ des golfeurs brassent des affaires tout en jouant au golf. Le golf de tournois et de vacances est un créneau qui prend de plus en plus d'ampleur en Estrie: il représente $28,3 \%$ des raisons de la pratique du golf.

Malgré la nature et l'environnement paisible des terrains de golf de l'Estrie, seulement $10.5 \%$ des golfeurs pratiquent ce hobby pour admirer le paysage. 
Les critères de sếlection des golfeurs pour le choix des terrains de golf

Un parcours de golf ayant une condition de terrain sans lacunes et un parcours ayant un excellent entretien général sont les aspects recherchés par près du tiers des golfeurs. Certains autres types de golfeurs $(22,8 \%)$ recherchent des caractéristiques précises (des éléments ponctuels) qui sortent de l'ordinaire comme par exemple des bons tertres de départ, de beaux verts, des allées pas trop usées, de bons golfeurs, etc.

Il n'est pas nécessaire qu'un terrain de golf soit difficile pour attirer les meilleurs golfeurs. Il n'y a que $16,7 \%$ des golfeurs qui recherchent un parcours de golf ardu. En fait l'accueil et le personnel en place (16,7\%) sont des éléments presqu'aussi importants que la compétitivité ellemême du parcours.

Le paysage et le décor $(6,8 \%)$, l'aceessibilite routiere $(5,0 \%)$, la longueur des parcours $(4,0 \%)$, et les activités complémentaires $(0,8 \%)$ sont d'autres critêres de selection quil sont plus ou moins importants lors de la recherche du terrain de golt,

\section{La distance à parcourir et les clubs de golf}

Les golfeurs en Estrie parcourent une très grande distance pour jouer au golf sur les terrains qui s'offrent à eux. Plus de $50 \%$ des golfeurs sont en mesure de parcourit plus de 50 kilomètres occasionnellement pour jouer au golf. En fait, $22.2 \%$ des adeptes font, de façon hebdomadaire, plus de 100 kilomètres. Parmi les plus mordus, quelques amateurs chevronnés $(5,9 \%)$ n'ont aucun problêne à parcourit' plus de 300 kilomètres pour aller frapper des petites balles blanches.

Les moins mordus du golf ( $23 \%$ des golfeurs) ne feraient pas plus de 25 kilometres pour pratiquer ce hobby.

\section{Le moment préféré dans}

la journée pour jouer au golf

Plus de la moitie des golfeurs en Estrie $(51,8 \%)$ préfèrent jouer au golf l'avantmidi (de 7 heures 11 heures). Ce moment est l'idéal pour eux parce qu' ils ont le restant de la journée pour faire autres choses. $20,5 \%$ des golfeurs préfèrent, quant à eux, jouer en après-midi. Les joueurs du midi ne représentent que $17 \%$ des golfeurs. Il est alors évident qu'il est plus facile pour un touriste de jouer à partir de 11 heures le matin. Les hôteliers qui offrent des forfaits doivent tenir compte de cette statistique car la grande majorité des golfeurs membres jouent le matin.

\section{Les golfeurs estriens à l'extérieur du Québec}

Les golfeurs estriens sont de grands consommateurs de clubs de golf étrangers: $62,4 \%$ des golfeurs estriens ont déja joué à l'extérieur du Québec. Sans que le golf en soit necessairement le motif principal, $41,1 \%$ de ces golfeurs ont réalisé 2 voyages ou moins (comprenant une partie de golf ou plus) au cours des 3 dernières années. $26,4 \%$ ont réalisé de 3 à 5 voyages tandis que $32,5 \%$ ont effectué plus de 6 voyages durant les 3 demières années.

L'endroit le plus visité des golfeurs qui ont joué a l'extérieur du Québec est la Floride avec $29,6 \%$ du total des voyages. Cette destination est surtout reconnue comme étant un lieu de villégiature hivernal des Québécois. Bien sûr, ces Québécois qui émigrent en Floride n'ont pas comme but premier de jouer au golf mais bénéficiant de vacances et d'un climat propice; ils en profitent pour jouer quelques parties. Par contre, les Carolines $(18,2 \%)$ et le Vermont $(15,8 \%)$ sont des destinations de golf si on peut les appeler ainsi. D'ailleurs, de nombreux voyages organisés en Estrie vont à Myrtle Beach en Caroline du Sud, la mecque des terrains de golf aux États-Unis. Plusieurs autres états américains sont populaires auprès des Estriens, devançant l'Ontario $(5,2 \%)$ et $1^{+}$Europe $(1,4 \%)$ pour les destinations de voyages.

$52,2 \%$ des voyages à l'étranger ont ét réalisés ayant pour motif principal le golf lui-même. Par contre la réalisation de parties de golf durant les vacances est un aspect tout aussi important. Plus de $42 \%$ des parties de golf à l'etranger se pratiquent durant les vacances. Les voyages d'affaires et de congrès sont très négligeables chez les golfeurs estriens. Ce taux ne représente que $5,4 \%$ des voyages à l'extérieur du Québec.

La qualite des terrains exterieurs est soit comparable $(43,1 \%)$ ou soit supérieure $(44,1 \%)$ par rapport à celle de l'Estrie.
Tableau 5

sMembershipo des clubs de golf de l'Estrie, 1996

\begin{tabular}{|l|c|}
\hline Nombre de membres & Nonbre de terrains \\
\hline 500 membres au plus & 1 \\
\hline Entre 400 et 499 membres & 7 \\
\hline Entre 300 et 399 membres & 7 \\
\hline Entre 200 et 209 membres & 7 \\
\hline Entru 100 et 199 membres & 9 \\
\hline Moins de 100 membres & 11 \\
\hline TOTAUX & 42 \\
\hline
\end{tabular}

Tableau 6

Les groupes d'age des golfeurs sur les parcours estriens, 199

\begin{tabular}{|c|c|}
\hline Groupe d'âge & Paurcentage \\
\hline Les juniars (17 ans at moins) & $6,9 \%$ \\
\hline Les jeunes menages (18 t 34 ans) & 20,18 \\
\hline Les menanes dutablis (35 aै 49 ans) & 30,35 \\
\hline Les bebes-boomers (50 aे 64 ans) & 28,58 \\
\hline Les retraites |65 ans et plus| & $11,2 \%$ \\
\hline TOTAUX & $100,0 \%$ \\
\hline
\end{tabular}

Tableau 7

Revenus annuels des golfeurs jouant en Estrie, 1995

\begin{tabular}{|c|c|}
\hline $\begin{array}{l}\text { Tranches de revenus } \\
\text { annuels des golfeurs }\end{array}$ & Representativite \\
\hline Mains de 150005 & $10,4 \mathrm{r}$ \\
\hline Entre 15001 et $30000 \mathrm{~S}$ & $27,1 \mathrm{~g}$ \\
\hline Entre 30001 et $50000 \$$ & $31,7 \%$ \\
\hline Entre 50001 et $90000 \$$ & $18,1 \%$ \\
\hline 80001 set plus & 12,7 午 \\
\hline TOTAUX & $100,0 \%$ \\
\hline
\end{tabular}

\begin{tabular}{|c|c|c|}
\hline \multicolumn{3}{|c|}{$\begin{array}{c}\text { Tablesu 8 } \\
\text { Qui joue sur lesgolfs de l'Estrie, } 1995\end{array}$} \\
\hline MRC de I'Estrie & $\begin{array}{l}\text { Nombre } \\
\text { de parties }\end{array}$ & $\begin{array}{l}\text { Aeprésen- } \\
\text { tativití: }\end{array}$ \\
\hline MAC de Sharbraoke & 228480 & $27,2 \%$ \\
\hline MAC de La Haute-Yamaska & 131040 & $15,6 \%$ \\
\hline MAC Memphrimagog & 52000 & $6.2 \%$ \\
\hline MRC de Caaticank & 36960 & 4,48 \\
\hline MAC Le Haut-Saint-François & 35532 & 4,28 \\
\hline MRC du Granit & 35028 & $4,2 \%$ \\
\hline MRC du Val-Saint-François & 33600 & $4,0 \%$ \\
\hline MAC Brome-Missisquai & 31920 & $3,8 \%$ \\
\hline MRC d'Asbestos & 17640 & $21 \%$ \\
\hline Hors Estris & 237695 & $28,3 \%$ \\
\hline TOTAUX & 840000 & $100,0 \%$ \\
\hline
\end{tabular}


Bien entendu, on ne peut comparer des terrains qui bénéfient de plus de 300 jours d'ensoleillement comme c'est le cas en Floride comparativement à 150 jours en Estric. Malgré cela, 11,4 \% des gens prétendent que les terrains de l'Estrie sont supérieurs dै ceux rencontrés durant les séjours à l'extérieur.

$26,3 \%$ des voyages extérieurs n'ont pas impliqué de nuitée. On peut en conclure que les voyages de golf ont été effectués dans les états frontaliers du Québec, soit dans l'état de New-York, du Vermont, du New-Hampshire ou du Maine. Le tiers des golfeurs estriens ont passé plus de 15 nuitées à l'étranger au cours des 3 dernières années. On peut supposer que les vacanciers en Floride se situent dans cette catégoric.

En 1997, $44 \%$ de l'ensemble des golfeurs iront au moins une fois à l'extérieur de l'Estrie pour jouer au golf. Près de $55 \%$ de ce nombre retoumeront aux États-Unis.

\section{Les tournois de golf}

Plus des deux tiers des joueurs de golf, soit $67,5 \%$, ont participé au moins à̀ un tournoi au cours de la demière année. Parmi ceux qui jouent lors de tournois, près de la moitié $(47 \%$ ) participent à trois tournois ou moins durant la saison de golf. Il y a $23,6 \%$ de smaniaques $*$ de tournois, c'est-à-dire ceux qui font plus de 7 tournois annuellement.

La MRC de La Haute-Yamaska est la MRC en Estrie qui accueille de loin, le plus de participants à des toumois de golf.

Les trois MRC qui accueillent le plus de joueurs-tournoi sont celles qui ont le moins d'habitants par terrain de golf dans leur région. Ces régions offrent une grande ouverture face à une clientèle extérieure (hor's Estrie).

\section{LES CARACTÉRISTIQUES DES TERRAINS DE GOLF DE L'ESTRIE}

Depuis les 3 dernières années, beaucoup de nouveaux terrains de golf ont été crés en Estrie. Parmi ceux-ci, il $y$ a entre autres le parcours du Vieux village à Bromont, un terrain comprenant 18 trous.

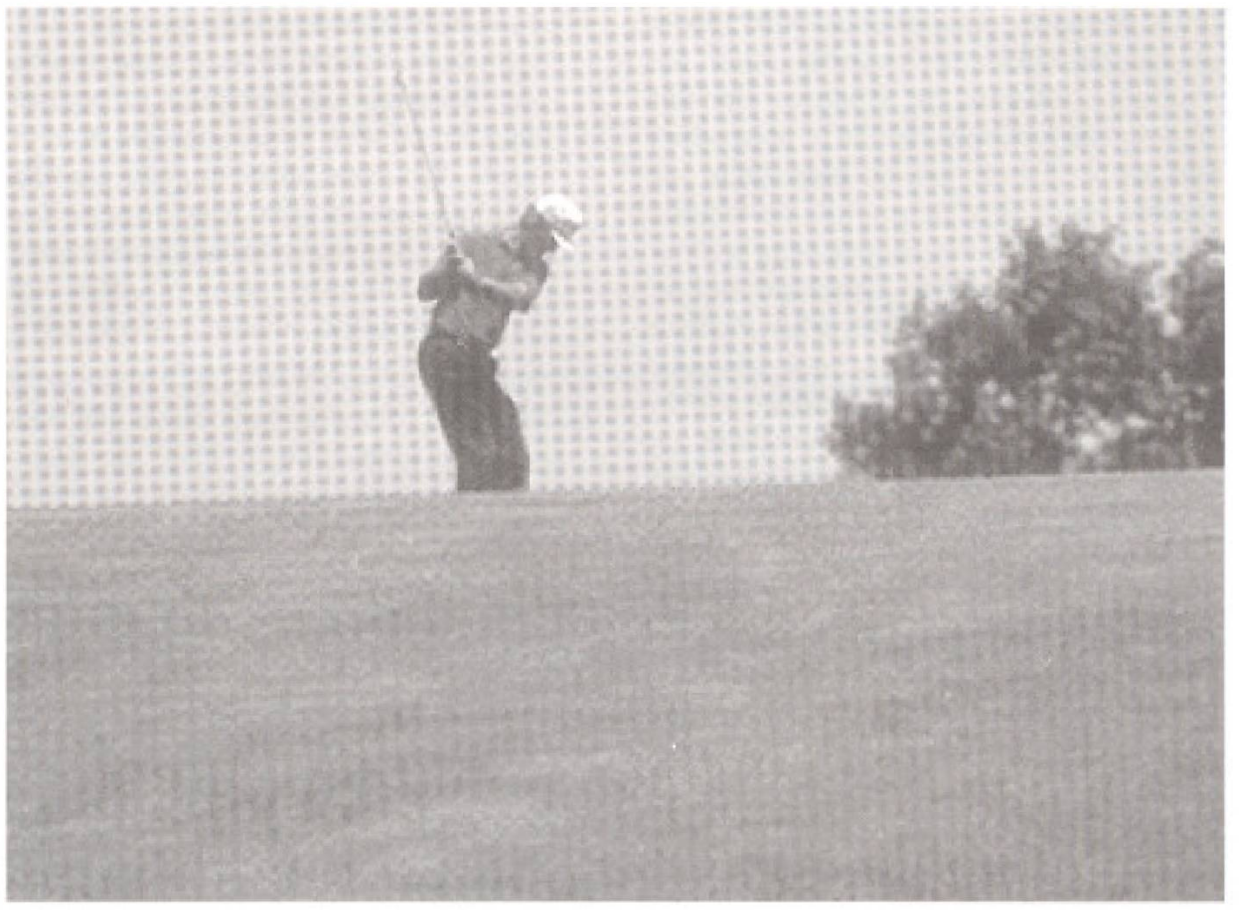

situé à proximité du Club de golf Royal Bromont, lequel ne date de pas plus de 5 ou 6 ans. Egalement a Bromont, près du parc industriel régional, il y a eu l'ouverture du Club de golf des Lacs comportant 18 trous de golf exécutif.

A cing minutes du club de Golf Inverness à Lac Brome, les travaux d'aménagement sont en cours pour la création d'un nouveau 18 trous. Même des terrains de golf établis depuis des dizaines d'années ont procédé à des phases d'agrandissement. On n'a qu'à penser au club de golf de Waterloo, à celui de Dufferin-Heights à Standstead, à celui du Lac Lyster à Baldwin Mills ou au club de golf du Manoir des Sables au Canton de Magog. La petite municipalitế d'East Angus a réussi à doubler son nombre de trous de golf en 1993 passant de 9 à 18. Avant le dépôt de ce mémoire, il y a eu ouverture de deux parcour's de normale 3: I'un étant à Granby et l'autre étant à Saint-Élied'Orford, Ces agrandissements et ouvertures de terrains ne se produisent pas seulement en Estrie. Le phénomène est encore plus grand en Montérégie où le nombre de terrains est maintenant de 61 . Cette région compte une proportion de $17 \%$ de l'offre québécoise totale. Dans les Laurentides, le Mont-Tremblant a réussi à créer un des plus beaux 18 trous au Québec en l'espace de 3 ans. Ces gestionnaires espèrent même créer 18 nouveaux trous d'ici l'an 2000.
La mauvaise distribution des terrains de golf en Estrie fait en sorte qu'il semble y avoir trop de terrains de golf sur le territoire, mais en même temps, il manque deux terrains de golf exécutifs (qui ne contient que des trous à normale 3 ou 4), soit dans la région de Magog-Orford et dans la région de Sherbrooke.

Malgré les 42 terrains à la disposition des golfeurs en Estrie, il n'y a que 9 terrains qui sont caractérisés de plats. A cause du relief du territoire de l'Estrie, les terrains de golf font face à deux grandes démarcations topographiques soit les grandes plaines du Saint-Laurent et le plateau appalachien. Ces terrains de golf sont situés à l'extrémité ouest de l'Estrie touristique soit sur les grandes plaines du Saint-Laurent. Cette topographie est plus favorable aux golfeurs moins en forme à cause du relief un peu plus plat. On retrouve entre autres les terrains tels Cowansville, Farnham et le Granby-StPaul.

Plus de $71 \%$ des golfeurs affirment qu'ils jouent aux heures qui leur conviennent. Cette statistique démontre bien que le produit de golf n'est plus aussi segmenté qu'auparavant.

Trois terrains de golf se démarquent en Estrie par la qualité générale du terrain. En effet, Les clubs de golf Owl's Head $(20,5 \%)$. Milby $(14,4 \%)$ et Cowansville 
$(12,9 \%)$ sont consideres par pres de la moitié des golfeurs comme ćtant les meilleurs terrains de l'Estrie. En fait $26,4 \%$ des golfeurs en Estrie prétendent que cette région est la plus belle au Québec en matière de golf. Ils reconnaissent qu'il y a de beaux parcours à l'extérieur de l'Estrie: Laval-sur-le-Lac $(7,6 \%)$, Royal Montréal $(6,8 \%)$, Joliette $(4,2 \%)$, Carling Lake $(4,2 \%)$, Le Manoir Richelieu $(4,2 \%)$ et I'Elm Ridge $(4,2 \%)$ ont eté identifies par les golfeurs en Estrie comme étant les meilleurs parcours extérieurs de l'Estrie.

\section{LES TOURISTES ET LE GOLF}

Le lien entre les terrains de golf et les entreprises touristiques est nébuleux. Théoriquement, il devrait y avoir de l'harmonic pour inciter les gens à jouer au golf et à faire d'autres activites pour qu'ils restent en Estric plus d'une jouméc. Le golf devrait etre l'activité idéale pour inciter les touristes a héberger en Estrie parce que le golf prend une plage horaire minimale de 5 heures dans la journée du joueur en plus des déplacements. Bref, les forfaits sont presque totalement méconnus par les touristes. L'Estrie se retrouve devant une industrie qui rapporte des millions de dollars et personne ne semble vouloir reconnaître et s'occuper de ce produit.

Certains terrains de golf de l'Estrie dépendent énormément de la présence des touristes dans leur's clubs. En effet. $25 \%$ des terrains de goll vivent exclusivement d'une clientèle extérieure. $50 \%$ ou plus des golfeurs proviennent de l'extéricur de l'Estrie. $18 \%$ des tertains ont d'excellents revenus d'appoint, obtenus put des visiteurs. Entre $25 \%$ et $50 \%$ des parties totales sont jouées par' les golfeurs touristiques et excursionnistes.

Il y a 23 terrains de golf en Estrie qui sont classés régionaux, c'est-à-dire que la clientèle extérieure représente moins de $20 \%$ de l'ensemble de leur clientèle (voir tableau 10).

- Les MRC de La Haute-Yamaska, Brome-Missisquoi et Memphrémagog reçoivent $87 \%$ du total des golfeurs extérieurs à l'Estrie. La Haute-Yamaska el Brome-Missisquoi recoivent généralement une clientèle excursionniste (aller-retour) étant donné un manque flagrant d"hébergement ainsi que la proximité de la grande région de Montréal. La région de Memphrémagog possède une clientèle touristique (qui reste apres le golf) car elle compte des attraits touristiques (lac Memphrémagog et mont Orford) ainsi que de l'hébergement à offrir en grande quantité (chalets, hôtels, motels, condos, etc.).

- Le taux d'occupation élevé par des joueurs locaux de la MRC de Sher= brooke limite l'accessibilité à̀ la clientèle extérieure.

Lorigine de la elientele extérieure (voir tableau 11):

- $75 \%$ des parties de golf jouées par les visiteurs proviennent de la grande région de Montréal ainsi que de sa rive-sud.

- Le club de golf l'Excellence d'Asbestos est très fréquenté par les gens de la région du Coeur-du-Québec étant donné la proximite de cette région.

- La clientèle hors Canada est surtout composée d'Américains qui sont situés non loin des frontières canadiennes. Les clubs de golf qui reçoivent le plus d'Améticains sont DufferinHeights, Lyster et Owl's Head, les trois clubs les plus au sud du Québec.

- La fréquentation des clubs de golf estriens par la clientèle extérieure est intimement liée par la qualité et la fluidité du réseau routier supérieur. En fait. la présence de l'autoroute 10 provoque un achalandage extérieur est-ouest beaucoup plus facile qu'une direction nord-sud. Etant situéc à l'extrémité sud-est de la province, l'Estrie ne possede pas une clientèle de transit comme la région du Coeur-du-Québec par exemple. C'est pour cette raison qu'il y a une faible représentativité de clientèle touristique de la région de la Gaspésie, de la Cote-Nord, du Saguenay-Lac-Saint-Jean et du Bas-SaintLaurent.

Les motifs de déplacement des joueurs de golf excursionnistes et touristiques en Estrie

Le golf n'est pas nécessairement le motif principal des déplacements de la clientèle exterieure. Les deux motifs principaux sont en fait la visite de parents et amis

\begin{tabular}{|c|c|}
\hline \multicolumn{2}{|c|}{$\begin{array}{c}\text { Tableau } 9 \\
\text { Classes de dipenses des golfeurs jouant en Estrie, } 1995\end{array}$} \\
\hline Categorie & Repressentativité \\
\hline Mains de $100 \mathrm{~S}$ & $7.3 \%$ \\
\hline Entre la1 3 at 250 3 & 10́. $11 \%$ \\
\hline Entre 251 s at $500 \mathrm{~s}$ & 20.35 \\
\hline Entre Sal $\$$ at $750 s$ & 15.25 \\
\hline Entre 751 \$ et 1000 \$ & 1264 \\
\hline Entre 1001 Set 20005 & 25. $3 x$ \\
\hline Plus de $2000 \mathrm{~s}$ & $9.2 \%$ \\
\hline TOTAL & $100,0 \%$ \\
\hline
\end{tabular}

\begin{tabular}{|c|c|}
\hline \multicolumn{2}{|c|}{$\begin{array}{c}\text { Tableau } 10 \\
\text { Importance des joueurs extraterritariaux } \\
\text { sur les golts de l'Estrie, } 1995\end{array}$} \\
\hline MAC de l'Esirie & $\begin{array}{l}\text { Nombre de parties } \\
\text { joutes par les visiteurs }\end{array}$ \\
\hline Haute-Yanaska & 91050 \\
\hline Memphrémagag & 74900 \\
\hline Brane-Missisquoi & 40950 \\
\hline Shertraoke & 15620 \\
\hline Coaticook & 7000 \\
\hline Ashestos & 2300 \\
\hline Lo Val-Stint-François & 2175 \\
\hline Le Haut-Saint-François: & 2100 \\
\hline Le Granit & 1600 \\
\hline TOTAUX & 237595 \\
\hline
\end{tabular}

$(24,9 \%)$ ainsi que les vacances en région $(23,1 \%)$. Par contre, étant en région, ces visiteurs en profitent pour jouer quelques parties de golf.

L'accessibilite et la beauté sont les motifs principaux reliếs au golf. $17,9 \%$ des visiteurs signalent que l'Estrie se démarque par son environnement et son faible taux d'occupation par rapport à lá grande région de Montréal.

Les tournois en région sont les motils de déplacement de $8,5 \%$ des visiteurs. D'autres raisons telles la réputation des tertains $(5,1 \%)$, la proximité du domicile $(8,5 \%)$, le rapport qualite-prix $(3,4 \%)$ et les retours aux lieux d'origines $(4,3 \%)$ sont tous des incitatifs qui amènent quelques touristes de plus durant la saison de golf.

\section{Les types d'hébergement prếconisés} par les golfeurs provenant de l'extérieur

Exceptế le quart de la clientèle extérieure qui fait des aller-retours, il y a 3 grands 


\begin{tabular}{|c|c|c|c|}
\hline \multicolumn{4}{|c|}{$\begin{array}{c}\text { Tableas } 11 \\
\text { Origine de la clientèle extérieure sur les golls de I'Estrie, } 1995\end{array}$} \\
\hline Clientele hors Estrie & Nombre de partiess & $\begin{array}{l}\text { Pourcentage des parties } \\
\text { totales en Estrie }\end{array}$ & $\begin{array}{l}\text { Pourcentage des } \\
\text { parties exterieures }\end{array}$ \\
\hline Montérégie & 97430 & 11,6 笪 & $41,0 \%$ \\
\hline MU1, Laval, Lanaudierre & 80685 & $9.6 \%$ & $33,9 \%$ \\
\hline Coeur-du-Québec & 21000 & $2,5 \%$ & $8,8 \%$ \\
\hline Les Laurentides ot l'Outaouais & 10915 & $1,3 \%$ & $5,0 \%$ \\
\hline Québec et envirans & 5880 & $0,7 \%$ & $2,5 \%$ \\
\hline Ailleurs au Quebbec & 11755 & $1,4 \%$ & $4,9 \%$ \\
\hline Ailleurs au Canada & 4200 & $0,5 \%$ & $1,8 \%$ \\
\hline Hors Canada & 5860 & $0,7 \%$ & $1,8 \%$ \\
\hline TOTAUX & 237695 & $28,3 \%$ & $100,0 \%$ \\
\hline
\end{tabular}

types d'hébergement qui sont préconisés par les touristes. Les chalets $(28,4 \%)$, les hotels-motels $(26,7 \%)$ et les nuitées chez les parents et amis $(14,7 \%)$ sont les plus populaires en Estrie. D'autres types d'hébergement telles les auberges, les gites du passant, les camping et autres ne représentent que $5,1 \%$ des nuitées de la part des touristes golfeurs.

\section{RECOMMANDATIONS}

1- Sachant fort bien qu'elle répond primordialement aux besoins de ses membres, I'Association touristique régionale de l'Estrie devrait s'occuper davantage de l'ensemble du produit de golf. Parce que le produit amène plus de 237000 parties par la clientèle extérieure par saison de golf malgré les lacunes des forfaits, il serait important de créer un comité de golf réunissant l'ensemble des terrains de golf de l'Estrie.

2- Il devrait y avoir des liens directs entre les bureaux touristiques locaux et les terrains de golf régionaux. Près de 213000 personnes s'y arrêtent en étét pour y prendre de l'information. Cette clientèle spotentiellew serait tentée si le produit de golf était clairement offert.

3- Sans vouloir discréditer les associations québécoises et canadiennes de golf, la région de la Montérégie, de Montréal et de Laval tirent assez fort sur la couverte de leurs associations respectives de golf de sorte que les autres régions du Québec sont oubliées. La région de $1^{\dagger}$ Estric serait bien positionnée quant à une nouvelle association de golf réunissant seulement les clubs de l'Estrie. Les cotisations de $1^{+}$Association estrienne serviraient:

- à promouvoir uniquement le golf comme produit d'appel de la région;

- à aller chercher des commanditaires en vue de quelques tournois majeurs se tenant sur le territoire estrien;

- à s"associer avec des hôteliers qui offriraient plus que des rabais ridicules de forfaits d'environ $10 \%$;

- à créer des ententes avec des clubs de golf dans quelques régions touristiques du Québec. Les mordus de golf étant des routiers à la recherche de consommation différente seraient comblés à tous points de vue. Par exemple, le Milby pourrait échanger avec le Grand-Mère, une réservation annuelle entre clubs membres comprenant par exemple 300 départs annuellement amènerait des retombées touristiques et économiques beaucoup plus intéressantes pour les deux régions concernées;

- à vendre le produit de golf estrien aux Américains en mettant en évidence le golf et l'ensemble de la région touristique, Avec un taux de change favorable, les Américains seraient moins hésitants ât visiter la région tout en essayant quelques parcours durant leur séjour.
4- Tout comme le ski à l'heure, il serait peut-être envisageable de créer un tarif par 9 trous. Les parcours à 9 trous ont tendance à devenir des 18 trous un peu plus tard. Certaines personnes (surtout les gens âgés) n'ont plus la capacité physique de jouer pendant plus de 4 heures sur les terrains de golf. La possibilité de jouer 9 trous sur les 18 présents serait un incitatif considérable. Cependant, cette possibilité augmenterait la charge des maréchals qui devraient superviser les entrées et les sorties de jeu plus fréquemment.

\section{NOTES}

I Profil de la elientête touristique québécoise en 1990. Produit touristique agolf" aw Québec. Février 1992.

2 Le golf au Québec, vert comme des dollars. Le Journal de Monréal, le 20 juillet 1990.

\section{BIBLIOGRAPHIE}

Giasson. François (1997) Profils des golfeurs et des terrains de golf en Esrie. Mémoire de man̂trise, Département de géographie et télédétection. Université de Sherbrooke, Sherbrooke, $300 \mathrm{p}$. 